\title{
Switch-on dynamics of nanocavity laser devices
}

\author{
Lorke, Michael; Nielsen, Torben Roland; Mørk, Jesper
}

Published in:

Applied Physics Letters

Link to article, DOI:

$10.1063 / 1.3651765$

Publication date:

2011

Document Version

Publisher's PDF, also known as Version of record

Link back to DTU Orbit

Citation (APA):

Lorke, M., Nielsen, T. R., \& Mørk, J. (2011). Switch-on dynamics of nanocavity laser devices. Applied Physics Letters, 99(15), 151110. https://doi.org/10.1063/1.3651765

\section{General rights}

Copyright and moral rights for the publications made accessible in the public portal are retained by the authors and/or other copyright owners and it is a condition of accessing publications that users recognise and abide by the legal requirements associated with these rights.

- Users may download and print one copy of any publication from the public portal for the purpose of private study or research.

- You may not further distribute the material or use it for any profit-making activity or commercial gain

- You may freely distribute the URL identifying the publication in the public portal

If you believe that this document breaches copyright please contact us providing details, and we will remove access to the work immediately and investigate your claim. 


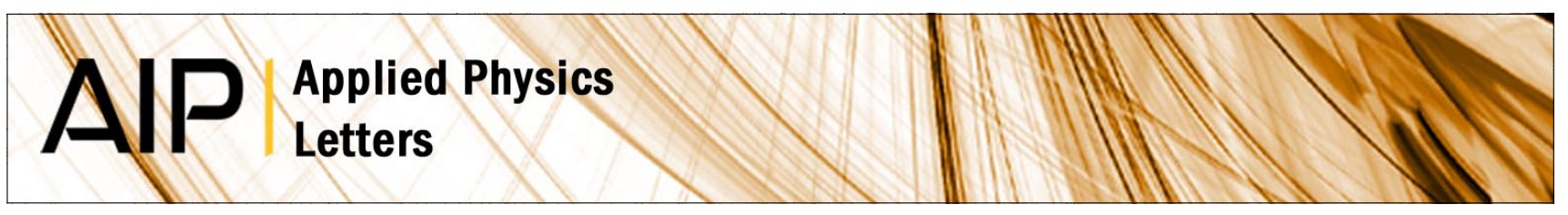

\section{Switch-on dynamics of nanocavity laser devices}

M. Lorke, T. R. Nielsen, and J. Mørk

Citation: Appl. Phys. Lett. 99, 151110 (2011); doi: 10.1063/1.3651765

View online: http://dx.doi.org/10.1063/1.3651765

View Table of Contents: http://apl.aip.org/resource/1/APPLAB/v99/i15

Published by the American Institute of Physics.

\section{Related Articles}

Near-field dynamics of broad area diode laser at very high pump levels AIP Advances 1, 042148 (2011)

Photonic bandstructure engineering of $\mathrm{THz}$ quantum-cascade lasers

Appl. Phys. Lett. 99, 201103 (2011)

Simulation of quantum cascade lasers

J. Appl. Phys. 110, 093109 (2011)

Ultra-broadband heterogeneous quantum cascade laser emitting from 2.2 to $3.2 \mathrm{THz}$

Appl. Phys. Lett. 99, 191104 (2011)

Wide range tuning of external cavity bent-waveguide laser diode

J. Appl. Phys. 110, 093105 (2011)

\section{Additional information on Appl. Phys. Lett.}

Journal Homepage: http://apl.aip.org/

Journal Information: http://apl.aip.org/about/about_the_journal

Top downloads: http://apl.aip.org/features/most_downloaded

Information for Authors: http://apl.aip.org/authors

\section{ADVERTISEMENT}

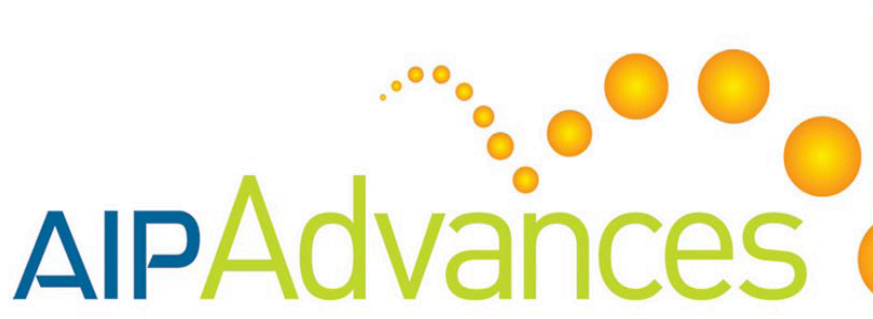

Submit Now

Explore AIP's new

open-access journal

Article-level metrics now available

Join the conversation!

Rate \& comment on articles 


\title{
Switch-on dynamics of nanocavity laser devices
}

\author{
M. Lorke, ${ }^{\text {a) }}$ T. R. Nielsen, and J. Mørk \\ DTU Fotonik, Department of Photonics Engineering, Technical University of Denmark, Building 343, \\ 2800 Kgs. Lyngby, Denmark
}

(Received 12 July 2011; accepted 21 September 2011; published online 13 October 2011)

\begin{abstract}
Theoretical investigations of the switch-on behavior of semiconductor quantum dot based nanocavity laser devices are presented. From a microscopic treatment of the carrier-carrier and carrier-photon interaction, we find a fast switch-on of the laser device that is enabled by ultrafast carrier dynamics and heavily damped relaxation oscillations. We show that the timescales of the dynamics within the continuum states and the quantum dot states are strongly coupled and investigate the time dependence of the non-equilibrium scattering rates in detail. ( 2011 American Institute of Physics. [doi:10.1063/1.3651765]
\end{abstract}

Semiconductor lasers are central components of optical technologies. They are most prominently used in optical communications and optical data storage involving compact discs, digital versatile discs, and blu-ray discs. Recently, semiconductor active regions coupled with nanoscale optical resonator schemes have opened an additional level of miniaturization and facilitated the implementation of photonic integrated circuits. The advent of photonic crystals allows to fabricate high-quality cavities, ${ }^{1-4}$ which opens a multitude of possibilities for guiding and modifying the emission properties via Purcell enhancement of emission rates, ${ }^{4}$ and also allows to directly integrate nanocavities with optical waveguides. For such photonic circuits, nanolasers are an important ingredient. The need for ever-higher data transmission rates calls for an increase in the modulation speed of the underlying laser devices. A fast switch-on behavior is a key quantity towards the realization of this goal, as unfavorable switch-on characteristics significantly limit the maximum possible transmission rate.

In recent years, semiconductor quantum dots (QDs) have proven to be an interesting system, as they possess a discrete energy spectrum that can be engineered to a large extent. They are considered as gain material for next generation optoelectronic devices and for fundamental studies of light-matter interaction. ${ }^{5-7}$

For the switch-on and modulation properties of QD laser devices, carrier scattering is a key quantity. Previous investigations on carrier scattering ${ }^{8}$ and its influence of the dynamical properties of QD lasers ${ }^{9-11}$ were based on quasi-equilibrium evaluations or rate equation analysis. In contrast, we present in this letter a full non-equilibrium analysis of the switch-on properties of QD-based nanolaser systems, including an analysis of carrier heating and non-equilibrium scattering rates.

We consider an ensemble of InGaAs QDs embedded in an optical nanocavity. Taken into account are the two lowest confined shells for electrons and holes, that are labeled sand p-shell, respectively, as well as the continuum of wetting layer (WL) states. Within the cluster expansion scheme, we derive coupled equations of motion for quantities like the photon number in the cavity mode and the carrier population of the different QD and WL states, extending previous work

\footnotetext{
a) Electronic mail: milo@fotonik.dtu.dk.
}

that focused on the QD states and carrier-photon correlations. ${ }^{12}$ It should be noted that, contrary to other investigations, ${ }^{9,10}$ we focus on nanocavity devices with a well separated mode spectrum and strong Purcell enhancement. The equations for the population of the state $v$ (QD or WL) and for the photon number in the mode $q$ are

$$
\begin{gathered}
\hbar \frac{\mathrm{d}}{\mathrm{d} t} f_{v}^{\lambda}=-2 \operatorname{Re} \sum_{q}\left|g_{q v}\right|^{2}\left\langle b_{q}^{\dagger} v_{v}^{\dagger} c_{v}\right\rangle \\
+S_{v, \text { Corr }}^{\lambda}+\left(1-f_{v}^{e}-f_{v}^{h}\right) P_{v}, \\
\left(\hbar \frac{\mathrm{d}}{\mathrm{d} t}+2 \kappa_{q}\right) n_{q}=+2 \operatorname{Re} \sum_{v^{\prime}}\left|g_{q v^{\prime}}\right|^{2}\left\langle b_{q}^{\dagger} v_{v^{\prime}}^{\dagger} c_{v^{\prime}}\right\rangle .
\end{gathered}
$$

Here, $\kappa_{q}$ is the cavity loss rate, connected to the $Q$-factor, $g_{q v}$ is the light-matter coupling constant, and $P_{v}$ is the pump contribution for the state $v$. Both $n_{q}$ and $f_{v}^{\lambda}$ couple to the photon assisted polarization $\left\langle b_{q}^{\dagger} v_{v}^{\dagger} c_{v}\right\rangle$

$$
\begin{aligned}
& \left(\hbar \frac{\mathrm{d}}{\mathrm{d} t}+\kappa_{q}+\Gamma+i\left(\tilde{\varepsilon}_{v}^{e}+\tilde{\varepsilon}_{v}^{h}-\hbar \omega_{q}\right)\right) \\
& \quad \times\left\langle b_{q}^{\dagger} v_{v}^{\dagger} c_{v}\right\rangle=f_{v}^{e} f_{v}^{h}-\left(1-f_{v}^{e}-f_{v}^{h}\right) n_{q} \\
& \quad+\delta\left\langle b_{q}^{\dagger} b_{q} c_{v}^{\dagger} c_{v}\right\rangle-\delta\left\langle b_{q}^{\dagger} b_{q} v_{v}^{\dagger} v_{v}\right\rangle .
\end{aligned}
$$

While it has been shown that an accurate description of dephasing under lasing conditions requires a non-Markovian treatment of the carrier-carrier and carrier-phonon interaction mechanisms, ${ }^{13}$ we include its influence into an effective dephasing rate $\Gamma$, which is determined by the carrier density dependent fit to the full non-Markovian calculation. ${ }^{14} \mathrm{~A}$ full microscopical inclusion of these effects is expected to only give minor changes as the emission dynamics is not very sensitive towards dephasing.

The higher order correlation functions, $\delta\left\langle b_{q}^{\dagger} b_{q} c_{v}^{\dagger} c_{v}\right\rangle$ and $\delta\left\langle b_{q}^{\dagger} b_{q} v_{v}^{\dagger} v_{v}\right\rangle$, obey their own equations of motion, see, e.g., Ref. 12 for details.

The rate $S_{v, \text { Corr }}^{\lambda}$ includes carrier-carrier and carrierphonon scattering and is given by

$$
S_{v, \text { Corr }}^{\lambda}=-f_{v}^{\lambda} S_{v}^{\lambda, \text { out }}+\left(1-f_{v}^{\lambda}\right) S_{v}^{\lambda, \text { in }}-\frac{f_{v}^{\lambda}-F_{v}^{\lambda}}{\tau_{\text {phonon }}^{\lambda}} .
$$


Here, the rates $S_{v}^{\lambda, \text { in,out }}$ for the carrier-carrier Coulomb interaction are evaluated in second order Born approximation and can be written as

$$
\begin{aligned}
S_{v}^{\lambda, \text { in }}= & \frac{2}{\hbar} \sum_{\mu v_{2} v_{3} v_{1}} W_{v v_{2} v_{3} v_{1}}\left(W_{v v_{2} v_{3} v_{1}}^{*}-W_{v v_{2} v_{1} v_{3}}^{*}\right) \\
& \times f_{v_{1}}^{\lambda}\left(1-f_{v_{2}}^{\mu}\right) f_{v_{3}}^{\mu} \pi \delta\left(\tilde{\varepsilon}_{v}^{\lambda}-\tilde{\varepsilon}_{v_{1}}^{\lambda}+\tilde{\varepsilon}_{v_{2}}^{\mu}-\tilde{\varepsilon}_{v_{3}}^{\mu}\right)
\end{aligned}
$$

and

$$
\begin{aligned}
S_{v}^{\lambda, \text { out }}= & \frac{2}{\hbar} \sum_{\mu v_{2} v_{3} v_{1}} W_{v v_{2} v_{3} v_{1}}\left(W_{v_{2} v_{3} v_{1}}^{*}-W_{v v_{2} v_{1} v_{3}}^{*}\right) \\
& \times\left(1-f_{v_{1}}^{\lambda}\right) f_{v_{2}}^{\mu}\left(1-f_{v_{3}}^{\mu}\right) \pi \delta\left(\tilde{\varepsilon}_{v}^{\lambda}-\tilde{\varepsilon}_{v_{1}}^{\lambda}+\tilde{\varepsilon}_{v_{2}}^{\mu}-\tilde{\varepsilon}_{v_{3}}^{\mu}\right),
\end{aligned}
$$

where the first and second terms in the square brackets correspond to direct and exchange Coulomb scattering, respectively. The matrix elements of the screened Coulomb interaction, $W_{v v_{2} v_{3} v_{1}}$, are calculated as in Ref. 8. The $\delta$-functions describe energy conservation in the Markov limit. NonMarkovian corrections to the carrier scattering are expected to give only small changes on the timescales considered here, see, e.g., Fig. 2 in Ref. 15 for the influence of nonMarkovian corrections for the example of the carrier-phonon interaction.

It is known that the carrier-phonon interaction needs to be treated within the polaron picture in QD systems. ${ }^{15}$ As the theoretical framework that is applied here for the light-matter interaction does not allow for an easy inclusion of polaron effects, we use a relaxation time approximation, where the quasi-equilibrium distributions for electrons and holes $F_{v}^{\lambda}$ are determined by the respective carrier densities in the system at a lattice temperature of $300 \mathrm{~K}$. The relaxation times $\tau_{\text {phonon }}^{\lambda}$ have been determined from independent polaron calculations and are taken to be 5 ps and 4 ps for electrons and holes, respectively. This approximation is only valid, if the carrierphonon scattering is not the dominant scattering mechanism, an assumption that is fulfilled in the regime of high excitation densities considered here. It should be noted that contrary to often made quasi-equilibrium assumptions for the continuum states, ${ }^{10,22}$ in this letter the full non-equilibrium dynamics of QD and WL states is included. This microscopic theory allows us to calculate dynamical properties of QD based laser devices

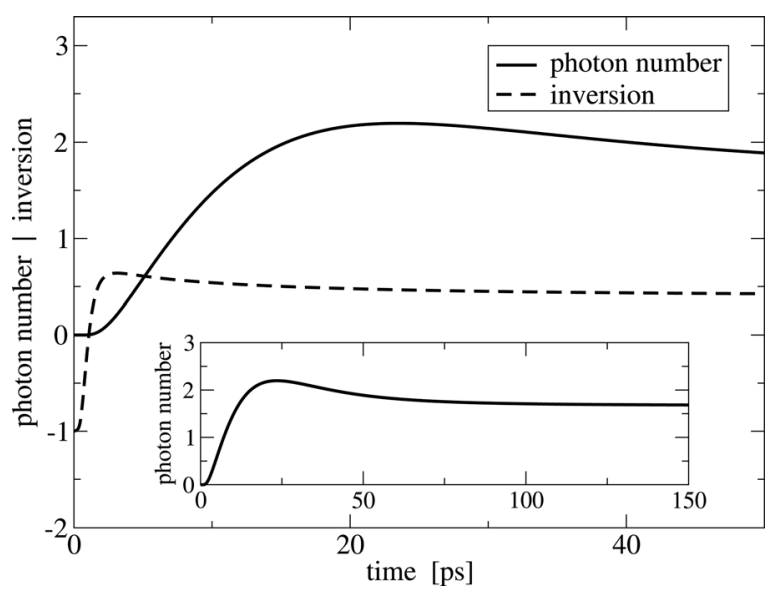

FIG. 1. Photon number and inversion of the QD s-shell transition as a function of time. The inset shows the long-time behavior of the photon number. such as modulation response ${ }^{16}$ and switch-on dynamics. In this work, we focus on the switch-on dynamics, as the switchon delay is an important parameter for large signal modulation. ${ }^{17}$

The results presented in Fig. 1 show the switch-on behavior of a nanolaser with a Q-factor of 2000 and a Purcell enhancement of $\mathrm{F}=20$, after optical $\mathrm{cw}$ excitation into the WL states, starting at $t=0$. The Purcell enhancement enters the light-matter coupling, as discussed in Ref. 12, and an rate equation analysis of the effect of Purcell enhancement on the modulation response of nanocavity devices can be found in Ref. 18. The level spacings for the QD states are taken as $40 \mathrm{meV}$ and $15 \mathrm{meV}$ for electrons and holes, respectively. The sheet density of QDs is $10^{10} / \mathrm{cm}^{2}$.

We observe a fast switch-on of the nanolaser, even though both capture into the QDs as well as relaxation within the QD needs to take place before emission can occur from the s-shell transition of the QDs. The photon number reaches a value of one, and stimulated emission dominates over spontaneous emission, after about 7 ps. The switch-on is accompanied by strongly overdamped relaxation oscillations, where only a single half-cycle is observed. This behavior is different from what has been observed in standard laser devices. ${ }^{10}$ The short timescale of the switch-on is consistent with experimental results on QW-based photonic-crystal lasers ${ }^{19}$ although we would like to stress that the results are not directly comparable due to the different gain materials. The inset of Fig. 1 shows the long time behavior of the photon number. It reveals that a steady state is reached within about 100 ps.

The steady state value that is reached is mostly controlled by the Q-factor, associated with the photon loss rate. In contrast, it is the combination of carrier kinetics and Purcell factor that determines the slope of the photon number vs. time curve and thus the time needed to reach steady state. The former is also influenced by the QD level spacing, with a larger (smaller) level spacing in general leading to lower (higher) scattering rates.

The fast switch-on is enabled by an ultra-fast carrier dynamics, which is seen in the inversion that is shown as the dotted line in Fig. 1.

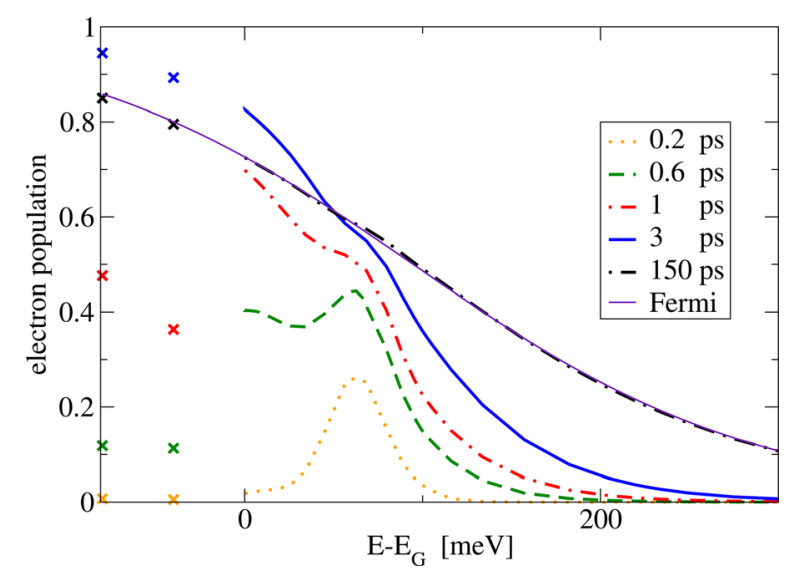

FIG. 2. (Color online) Electron population function as a function of energy for different times. Solid lines represent the WL populations and crosses the QD populations. The thin line is a quasi-Fermi distribution that is in excellent agreement with the steady-state result of the kinetics. 
The inversion reaches the maximum value after about 3 ps, which points towards extremely efficient carrier capture and relaxation. We also observe that there is little spectral hole burning (shb), as the inversion has a steady state value of $\approx 0.45$. This can be explained by the low photon number that is present in this device. Since the average photon number is only about 2 in steady state, the stimulated emission rate is not much larger than other rates in the system, as it would be in conventional laser devices, which would cause significant shb. In contrast, the rates for emission and carrier scattering for such a nanolaser device are of comparable order of magnitude, which means that the degree of shb observed depends on the details of the system. It should be noted that the switch-on behavior cannot be reproduced by using constant scattering times for the Coulomb interaction.

The details of the carrier dynamics are shown in Fig. 2, where the carrier population functions is shown as a function of energy for different times. After the initial Gaussian population profile excited by the pump, we observe an ultra-fast redistribution that immediately starts to populate the QD states. Remarkably, the timescales between the relaxation dynamics in the $\mathrm{WL}$ and the dynamics of the QD populations are not decoupled, as often assumed. ${ }^{10,22}$ On one hand, this is due to the efficiency of the initial in-scattering into the QD, as the QD states are initially empty. On the other hand, the relaxation within the WL is significantly slower than in a pure quantum well. The latter effect is caused by the fact that the capture into the QD states is most efficient for the WL states with low quasi-momenta. Therefore, these states are constantly depleted during the early stage of the kinetics, which slows down the relaxation of the WL distribution itself towards a quasiequilibrium distribution. Moreover, a common chemical potential for QD and WL states is reached, as it is expected from experimental data for temperatures over $250 \mathrm{~K}^{20}$

Furthermore, after the initial relaxation that lasts about 3 ps, a heating of the carrier population is observed on a timescale of $100 \mathrm{ps}$, as the pumping into the WL injects carriers at a higher energy than the emission energy. Even though the lattice temperature is held at $300 \mathrm{~K}$, the resulting steady-state Fermi distribution has a carrier temperature of $\approx 1120 \mathrm{~K}$. This effect has been observed in QW laser structures, ${ }^{21}$ but is more pronounced in QDs, as the energy difference between injected and recombining carriers is larger and has also been discussed for QD devices. ${ }^{22}$

In Fig. 3, we present the non-equilibrium scattering rates as functions of time. After an initial rise, the out-scattering rates both for electrons and holes exhibit a maximum at about 600/ps. The high rate is outbalanced by the low QD occupation (cf. Eq. (4)) during the early stage of the kinetic. After this fast initial rise, the out-scattering rates are reduced significantly and rise again only weakly in conjunction with the above mentioned heating. The in-scattering rates also increase strongly within the first picosecond, leading to the observed fast carrier dynamics. Moreover, there is a sharp increase in the in-scattering rate for the electrons, approximately at the time when the WL distribution for electrons first acquires significant values at the band edge.

In conclusion, we have demonstrated a fast switch-on of QD-based nanolaser devices that is driven by an ultra-fast carrier dynamics. The associated scattering rates exhibit a

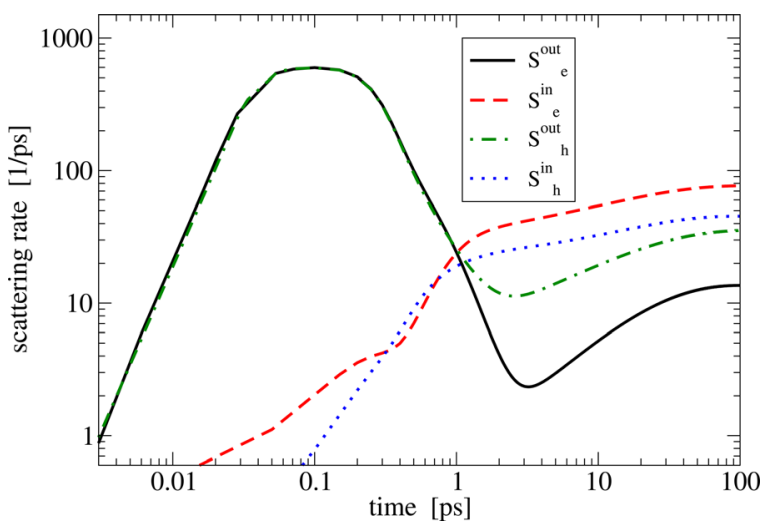

FIG. 3. (Color online) In-scattering and out-scattering rates of the QD s-shell for electrons and holes as a function of time.

very strong time dependence, whose inclusion is vital to describe the switch-on behavior. The combination of fast switch-on dynamics and weak relaxation oscillations shows that such laser structures are promising candidates for ultracompact on-chip signal sources.

M.L. acknowledges financial support from the Danish Research Council for Technology and Production and from the "Young Elite Researcher Award" initiative by the Danish Council for Independent Research. T.R.N. and J.M. acknowledge support from the Villum Kann Rasmussen Center "NATEC."

${ }^{1}$ M. Nishioka, R. Schur, M. Kitamura, H. Watabe, and Y. Arakawa, Physica B 227, 404 (1996)

${ }^{2}$ H. Saito, K. Nishi, I. Ogura, S. Sugou, and Y. Sugimoto, Appl. Phys. Lett. 69, 3140 (1996)

${ }^{3}$ K. Vahala, Nature 424, 839 (2003).

${ }^{4}$ P. Lodahl, A. Floris van Driel, I. S. Nikolaev, A. Irman, K. Overgaag, D. Vanmaekelbergh, and W. L. Vos, Nature 430, 654 (2004).

${ }^{5}$ M. Bayer, T. L. Reinecke, F. Weidner, A. Larionov, A. McDonald, and A. Forchel, Phys. Rev. Lett. 86, 3168 (2001).

${ }^{6}$ J. P. Reithmaier, G. Sek, A. Löffler, C. Hofmann, S. Kuhn, S. Reitzenstein, L. Keldysh, V. D. Kulakovskii, T. L. Reinecke, and A. Forchel, Nature 432, 197 (2004).

${ }^{7}$ T. Yoshie, A. Scherer, J. Hendrickson, G. Khitrova, H. M. Gibbs, G. Rupper, C. Ell, O. B. Shchekin, and D. G. Deppe, Nature 432, 200 (2004).

${ }^{8}$ T. Nielsen, P. Gartner, and F. Jahnke, Phys. Rev. B 69, 235314 (2004).

${ }^{9}$ W. Chow and S. Koch, IEEE J. Quantum Electron. 41, 495 (2005).

${ }^{10}$ K. Luedge, M. J. P. Bormann, E. Malic, P. Hoevel, M. Kuntz, D. Bimberg, A. Knorr, and E. Schoell, Phys. Rev. B 78, 035316 (2008).

${ }^{11}$ D. W. Reschner, E. Gehrig, and O. Hess, IEEE J. Quantum Electron. 45, 21 (2009).

${ }^{12}$ C. Gies, J. Wiersig, M. Lorke, and F. Jahnke, Phys. Rev. A 75, 013803 (2007).

${ }^{13}$ M. Lorke, T. Nielsen, J. Seebeck, P. Gartner, and F. Jahnke, Phys. Rev. B 73, 85324 (2006).

${ }^{14}$ M. Lorke, J. Seebeck, T. Nielsen, P. Gartner, and F. Jahnke, Phys. Stat. Sol. (c) 3, 2393 (2006).

${ }^{15}$ J. Seebeck, T. Nielsen, P. Gartner, and F. Jahnke, Phys. Rev. B 71, 125327 (2005).

${ }^{16}$ M. Lorke, T. R. Nielsen, and J. Mørk, Appl. Phys. Lett. 97, 211106 (2010).

${ }^{17}$ L. Coldren and S. Corzine, Diode Lasers and Photonic Integrated Circuits (Wiley, New York, 1995).

${ }^{18}$ T. Suhr, N. Gregersen, M. Lorke, and J. Mørk, Appl. Phys. Lett. 98, 211109 (2011).

${ }^{19}$ H. Altug, D. Englund, and J. Vučkuvić, Nat. Phys. 2, 484 (2006).

${ }^{20}$ I. O'Driscoll, P. Smowton, and P. Blood, IEEE J. Quantum Electron. 45, 380 (2009).

${ }^{21}$ F. Jahnke and S. W. Koch, Opt. Lett. 18, 1438 (1993).

${ }^{22}$ A. V. Uskov, C. Meuer, H. Schmeckebier, and D. Bimberg, Appl. Phys. Express 4, 022202 (2011). 